\title{
Properties of Discrete Dynamical System in BCl-Algebra
}

\author{
Dawood Khan", Abdul Rehman, Naveed Sheikh, Saleem Iqbal, Israr Ahmed
}

Department of Mathematics, University of Balochistan, Quetta, Pakistan

\section{Email address:}

dawooddawood601@gmail.com (D. Khan)

${ }^{*}$ Corresponding author

\section{To cite this article:}

Dawood Khan, Abdul Rehman, Naveed Sheikh, Saleem Iqbal, Israr Ahmed. Properties of Discrete Dynamical System in BCI-Algebra. International Journal of Management and Fuzzy Systems. Vol. 6, No. 3, 2020, pp. 53-58. doi: 10.11648/j.ijmfs.20200603.12

Received: September 16, 2020; Accepted: October 13, 2020; Published: November 11, 2020

\begin{abstract}
In the present manuscript we introduce the concept of some notions such as fixed points, periodic points, invariant set and strongly invariant or S-invariant set of discrete dynamical system $(Z, \Psi)$ in BCI-algebra where in $(Z, \Psi)$, Z is a nonempty set and supposed to be a BCI-algebra and the mapping $\Psi$ is a homomorphism from $Z$ to $Z$ and establish some new homomorphic properties of BCI-algebra based on these notions. We also prove some new results related to the set that contains the all fixed points and to the set that contains all periodic points in $\mathrm{Z}$ such that we prove that the set of all fixed points and the set of all periodic points in BCI-algebra $\mathrm{Z}$ are the BCI-sub algebras. We show that when a sub set of BCI-algebra Z is an invariant set with respect to $\Psi$. We prove that the set of all fixed points and the set of all periodic points in p-semisimple BCIalgebra $\mathrm{Z}$ are the ideals of $\mathrm{Z}$. We also prove that the set of all fixed points in $\mathrm{Z}$ is an S-invariant subset of a BCI-algebra Z. We have no doubt that the research along this line can be kept up, and indeed, some results in this manuscript have already made up a foundation for further exploration concerning the further progression of a discrete dynamical system in BCI-algebra and their applications in other disciplines of algebra.
\end{abstract}

Keywords: BCI-Algebra, Discrete Dynamical System, Periodic Points, Fixed Points, Invariant Set, S-invariant or (Strongly Invariant) Set

\section{Introduction}

The foundation of the concept of BCI-Algebra was laid down by the famous mathematician Iseki in his pioneering paper [9]. His theory about BCI-algebra and related ideas and properties are nowadays utilized extensively in different areas of science like artificial intelligence information sciences, cybernetics and computer sciences. Both BCKalgebra and BCI-algebra have been inspired by two considerations one based on classical and non-classical propositional calculi of Meredith and the other based on set theory [11]. The concept of ideal theory of BCI-algebra plays a fundamental role in the evolution of BCI-Algebra was first introduced by Iseki while the notion of BCI- homomorphism was also first defined by Iseki in [10]. During the past four decades several researchers have extensively investigated this field and have produced a lot of literatures about the theory of BCI-algebra $[8,13]$.

The foundation of dynamical system was laid down by the eminent mathematician Henri Poincare in 1899 in his famous paper celestial mechanics [7]. His theories about dynamical systems and connected ideas and properties are nowadays widely used in various fields of science like physics, biology, meteorology astronomy, economics and many others. The main idea of the study of dynamical system indicates the mathematical techniques for describing the eventual or asymptotic behavior of an iterative process in different specified scientific disciplines. In 1917 Gaston Julia and Pierre Fatau diverted the concept of the theory of dynamical system in relationship of complex analysis and established a new notion and provided the name as complex analytic dynamical system [6]. Latterly Birkhoff enthusiastically adopted the Poincare's viewpoint and realized the significance of the concept of mappings and introduced discrete dynamical system. Discrete dynamical system is an interesting and active area of applied and pure mathematics that involves tools and techniques from different fields such as Number Theory, Analysis and Geometry. Discrete dynamical systems are those dynamical systems whose states evolve over a state space in discrete steps according to a fixed rule [1]. Birkhoff's opinion based on discrete steps was 
highly captivating and attracted the attention of mathematician to use in various fields of mathematics. Many researchers applied the concept of discrete dynamical system in their related areas. This becomes the extension of the Theory of discrete dynamical system in many branches of mathematical sciences. In 1927 Birkhoff infused the notion of discrete dynamical system in topology and laid down the foundation of another field known as topological Dynamics [17]. Topological dynamics then flourished and further generated algebraic topology and differential topology [3]. Differential Topological techniques enabled piexoto and Smale to understand the chaotic behavior of a large class of dynamical systems and introduced a new area of dynamical system known as hyperbolic dynamical system [15, 19]. Von Neumann, Birkhoff and Koopman introduced discrete dynamical system in measure theory due to which a new filed emerged by the name of Ergodic theory [18]. Recently Dikranjan and Bruno embedded the concept of discrete dynamical system in group theory and established a new algebraic structure known as discrete dynamical systems in group theory [5]. Generalization in framework of discrete dynamical systems in group theory, Dikranjan Bruno provided the concept of Entropy on abedian groups [20] and Dardano et al. introduced the inertial properties in groups [4].

In the present paper, we emphasize the mathematical aspects of the theory of discrete dynamical system $(Z, \Psi)$. We define the concept of discrete dynamical system $(Z, \Psi)$ in BCI-algebra where $Z$ is a BCI-algebra and $\Psi$ is a homomorphism from $Z$ to $Z$ and establish some properties of the set of periodic points and the set of fixed points of BCIalgebra $Z$. We prove that the sets of all fixed points and periodic points of BCI-algebra Z are the BCI-sub algebras of $Z$. We show that when a subset of a BCI-algebra $Z$ is invariant with respect to $\Psi$. we prove that the sets of all fixed and periodic points of a p-semisimple $\mathrm{BCI}$-algebra $\mathrm{Z}$ are the ideals of $Z$. we also prove that in the discrete dynamical system $(Z, \Psi)$ the set of all fixed points is S-Invariant subset of a BCI-algebra $Z$.

\section{Preliminaries}

This section consists of some preliminary definitions and basic facts about BCI-algebra which are useful in the proofs of our results. Throughout this research work we denote the BCI-algebra always by Z without any specification. Here we only mention those concepts of BCI-algebra which are necessary for our treatment. For further information regarding BCI-algebra the readers are referred to [13].

Definition 2.1: [12] A BCI-algebra $(Z, *, 0)$ is a class of the type $(2,0)$ algebras satisfying the following four axioms for all $\mathrm{a}, \mathrm{b}, \mathrm{c} \in \mathrm{Z}$,

$$
\begin{array}{ll}
(\text { BCI-i). } & ((\mathrm{a} * \mathrm{~b}) *(\mathrm{a} * \mathrm{c})) *(\mathrm{c} * \mathrm{~b})=0 \\
\text { BCI-ii)• } & (\mathrm{a} *(\mathrm{a} * \mathrm{~b})) * \mathrm{~b}=0 \\
\text { (BCI-iii). } & \mathrm{a} * \mathrm{a}=0 \\
\text { (BCI-iv). } & \mathrm{a} * \mathrm{~b}=0 \text { and } \mathrm{b} * \mathrm{a}=0 \Rightarrow \mathrm{a}=\mathrm{b}
\end{array}
$$

Moreover $\leq$ is a partial order on $\mathrm{Z}$ defined by $\mathrm{a} \leq \mathrm{b} \Leftrightarrow \mathrm{a} *$ $\mathrm{b}=0$

Proposition 2.2: $[10,12]$ the following conditions are true in any BCI-algebra Z.
(C-i)
$(\mathrm{a} * \mathrm{~b}) * \mathrm{c}=(\mathrm{a} * \mathrm{c}) * \mathrm{~b}$
(C-ii) $\quad(a * c) *(b * c) \leq a * b$
$, \forall \mathrm{a}, \mathrm{b}, \mathrm{c} \in \mathrm{Z}$
(C-iii) $\quad(\mathrm{a} * \mathrm{~b}) *(\mathrm{a} * \mathrm{c}) \leq \mathrm{c} * \mathrm{~b}$
(C-iv)
$\mathrm{a} * 0=\mathrm{a}$
(C-v) $\quad \mathrm{a} *(\mathrm{a} *(\mathrm{a} * \mathrm{~b}))=\mathrm{a} * \mathrm{~b}$
(C-vi) $\quad \mathrm{a} \leq \mathrm{b} \Rightarrow \mathrm{c} * \mathrm{~b} \leq \mathrm{c} * \mathrm{a}$
(C-vii) $\quad 0 *(\mathrm{a} * \mathrm{~b})=(0 * \mathrm{a}) *(0 * \mathrm{~b})$
(C-viii) $\quad \mathrm{a} \leq \mathrm{b} \Rightarrow \mathrm{a} * \mathrm{c} \leq \mathrm{b} * \mathrm{c}$
$, \forall \mathrm{a}, \mathrm{b}, \mathrm{c} \in \mathrm{Z}$
$, \forall \mathrm{a}, \mathrm{b}, \mathrm{c} \in \mathrm{Z}$
,$\forall \mathrm{a} \in \mathrm{Z}$
$, \forall \mathrm{a}, \mathrm{b} \in \mathrm{Z}$
$, \forall \mathrm{a}, \mathrm{b}, \mathrm{c} \in \mathrm{Z}$
$, \forall \mathrm{a}, \mathrm{b}, \mathrm{c} \in \mathrm{Z}$
$, \forall \mathrm{a}, \mathrm{b}, \mathrm{c} \in \mathrm{Z}$

Definition 2.3: [12] Let Z_s be a non-vacuous subset of a BCI-algebra

$\left(Z_{,}, *, 0\right)$ then $Z_{\_} s$ is said to be a BCI-subalgebra of $Z_{\text {if }}$ it satisfies the (s-i) and (s-ii) conditions where
(s-i).
0 Z, Z_s
(s-ii).
$\mathrm{a} * \mathrm{~b} Z \mathrm{Z} Z \_s$ for any $\mathrm{a}, \mathrm{b} \in \mathrm{Z}$ _s

Definition 2.4: [12] Let $Z$ be a BCI-algebra and $I_{d}$ be a non-vacuous subset of $Z$ then $I_{d}$ is said to be an ideal of $Z$ if it satisfies $\left(I_{d}-\mathrm{i}\right)$ and $\left(I_{d}\right.$-ii) conditions, where

$$
\begin{array}{ll}
\left.I_{d}-\mathrm{i}\right) & 0 \in I_{d} \\
\left(I_{d}-\mathrm{ii}\right) & \mathrm{a} * \mathrm{~b} \in I_{d} \text { and } \mathrm{b} \in I_{d} \Rightarrow \mathrm{a} \in I_{d} \quad, \forall \mathrm{a}, \mathrm{b} \in \mathrm{Z}
\end{array}
$$

Definition 2.5: [10] A mapping $\Psi: Z_{1} \rightarrow Z_{2}$ where $Z_{1}$ and $Z_{2}$ are two BCI-algebras is said to be a BCI-homomorphism if it satisfies the following condition

$$
\Psi(\mathrm{a} * \mathrm{~b})=\Psi(\mathrm{a}) * \Psi(\mathrm{b}) \quad, \mathrm{a}, \mathrm{b} \in \mathrm{Z}_{1}
$$

Definition 2.6: [14] A BCI-algebra $(Z, *, 0)$ is psemisimple if

$(\forall \mathrm{a} \in \mathrm{Z})(0 * \mathrm{a}=0 \Longrightarrow \mathrm{a}=0)$.

Theorem 2.7: [2] If a BCI-algebra $(Z, *, 0)$ is p-semisimple and $a, b, c \in Z$ then

$\left(\mathrm{T}_{1}\right) \mathrm{c} * \mathrm{a} \leq \mathrm{c} * \mathrm{~b} \Rightarrow \mathrm{a}=\mathrm{b}$;

$\left(\mathrm{T}_{2}\right) \mathrm{a} * \mathrm{c} \leq \mathrm{b} * \mathrm{c} \Rightarrow \mathrm{a}=\mathrm{b}$;

Theorem 2.8: [2] If a BCI-algebra $(Z, *, 0)$ is p-semisimple and $a, b, c \in Z$ then

$\left(\mathrm{T}^{\prime}{ }_{1}\right) \mathrm{c} * \mathrm{a}=\mathrm{c} * \mathrm{~b} \Rightarrow \mathrm{a}=\mathrm{b}$;

$\left(\mathrm{T}_{2}^{\prime}\right) \mathrm{a} * \mathrm{c}=\mathrm{b} * \mathrm{c} \Rightarrow \mathrm{a}=\mathrm{b}$;

\section{Basic Definitions of Discrete Dynamical System in BCI-Algebra}

In this section we pick some terminologies of discrete dynamical system and define them in terms of BCI-algebra

Definition 3.1: Let $Z$ be a BCI-algebra and $\Psi: Z \rightarrow Z$ be a homomorphism then $(Z, \Psi)$ is called a discrete dynamical system in BCI-algebra

In the present paper whenever we say a discrete dynamical system it means we are taking an ordered pair $(Z$, $\Psi$ ) where $Z$ is a BCI-algebra and $\Psi$ is a homomorphism from $Z$, to $Z$.

Definition 3.2: In the discrete dynamical system $(Z, \Psi)$, a point $\mathrm{a} \in \mathrm{Z}$ is a fixed point if $\Psi(\mathrm{a})=\mathrm{a}$ 
Definition 3.3: In the discrete dynamical system $(Z, \Psi)$, a point $a \in Z$ is a periodic point if $\Psi^{m}(a)=a$ for some positive integer $m$, the least value of $m$ is said to be the period of "a"

Definition 3.4: In the discrete dynamical system $(Z, \Psi)$, a subset $A$ of $Z$ is an invariant subset of $Z$ if $\Psi(A) \subset A$.

Definition 3.5: In the discrete dynamical system $(Z, \Psi)$, a subset $A$ of $Z$ is a strongly invariant subset of $\Psi$ if $\Psi(A)=A$.

\section{Basic Results}

In this section we prove some properties which are essential in proving the theorems in this paper.

Proposition 4.1: If a mapping $\Psi: Z \rightarrow Z$ is a homomorphism from BCI-algebra $Z$, to $Z$ and $0 \in Z$, then $\Psi$ $(0)=0$.

$$
\begin{array}{ll}
\text { Proof:- } \Psi(0)=\Psi(0 * 0) & \because \text { BCI-iii } \\
=\Psi(0) * \Psi(0)=0 & \because \text { BCI-iii }
\end{array}
$$

Proposition 4.2: If $\Psi: Z \rightarrow Z$ is a homomorphism then $\Psi^{n}$ : $Z \rightarrow Z$ is a homomorphism. (Here $\Psi^{n}$ means $\Psi_{0} \Psi_{0} \Psi \ldots \circ \Psi$ (n time))

Proof: We prove this result by using the principle of mathematical induction. We have to show that for any two elements $\mathrm{a}, \mathrm{b}$ in $\mathrm{Z}$

$$
\Psi^{n}(\mathrm{a} * \mathrm{~b})=\Psi^{n}(\mathrm{a}) * \Psi^{n}(\mathrm{~b})
$$

Where $\mathrm{n}$ is the positive integer

It has been given that statement (1) is valid for $n=1$ and we assume that it is valid for $n=k$ then we have

$$
\begin{aligned}
& \Psi^{k}(\mathrm{a} * \mathrm{~b})=\Psi^{k}(\mathrm{a}) * \Psi^{k}(\mathrm{~b}) \\
& \Psi\left(\Psi^{k}(\mathrm{a} * \mathrm{~b})\right)=\Psi\left(\Psi^{k}(\mathrm{a}) * \Psi^{k}(\mathrm{~b})\right)
\end{aligned}
$$$$
\Psi^{k+1}(\mathrm{a} * \mathrm{~b})=\Psi\left(\Psi^{k}(\mathrm{a})\right) * \Psi\left(\Psi^{k}(\mathrm{~b})\right) \because \Psi \text { is homomorphism }
$$$$
\Psi^{k+1}(\mathrm{a} * \mathrm{~b})=\Psi^{k+1}(\mathrm{a}) * \Psi^{k+1}(\mathrm{~b})
$$

Thus the validity of statement (1) at $n=k$ implies the validity of (1) at $\mathrm{n}=\mathrm{k}+1$

Hence (1) is true for all positive integers $n$.

Proposition 4.3: If $\Psi: Z \rightarrow Z$ is a homomorphism, $\Psi^{n}(\mathrm{a})=\mathrm{a}$ and $\mathrm{n}, \mathrm{p}$ are positive integers such that $\mathrm{n}$ divides $\mathrm{p}$ then $\Psi^{p}(\mathrm{a})=\mathrm{a}$

$$
\text { Proof: Since } \Psi^{n}(\mathrm{a})=\mathrm{a}
$$

Where $\mathrm{n}$ is a positive integer which divides $\mathrm{p}$ then there exists an integer $\mathrm{q}$ such that $\mathrm{p}=\mathrm{n} \mathrm{q}$ then we have

$$
\begin{aligned}
& \Psi^{p}(\mathrm{a})=\Psi^{n q}(\mathrm{a})=\Psi^{n(q-1)}\left(\Psi^{n}(\mathrm{a})\right) \\
& =\Psi^{n(q-1)}(\mathrm{a}) \quad \because \operatorname{using}(2) \\
& =\Psi^{n(q-2)}\left(\Psi^{n}(\mathrm{a})\right) \\
& =\Psi^{n(q-2)}(\mathrm{a}) \\
& \vdots \\
& \vdots \\
& =\Psi^{n(q-(q-1))}(\mathrm{a})=\Psi^{n(q-q+1)}(\mathrm{a})=\Psi^{n}(\mathrm{a})=\mathrm{a}
\end{aligned}
$$

The following are simple examples regarding the definitions given in the paper. Example (1): suppose that $\mathrm{Z}=\{0, \mathrm{p}, \mathrm{q}, \mathrm{r}\}$ is a BCI-algebra
Table 1. Tabular arrangement of the values of the set satisfying the axioms of BCI-algebra.

\begin{tabular}{lllll}
\hline$*$ & $\mathbf{0}$ & $\mathbf{p}$ & $\mathbf{q}$ & $\mathbf{r}$ \\
\hline 0 & 0 & 0 & 0 & $\mathrm{q}$ \\
$\mathrm{p}$ & $\mathrm{p}$ & 0 & 0 & $\mathrm{r}$ \\
$\mathrm{q}$ & $\mathrm{q}$ & $\mathrm{q}$ & 0 & $\mathrm{r}$ \\
$\mathrm{r}$ & $\mathrm{r}$ & $\mathrm{r}$ & $\mathrm{r}$ & 0 \\
\hline
\end{tabular}

And a mapping $\Psi: Z \rightarrow Z$, defined by $\Psi(0)=0, \Psi(\mathrm{p})=0, \Psi(\mathrm{q})=0$ and $\Psi(\mathrm{r})=\mathrm{r}$

is a homomorphism. Then the points 0 and $r$, are fixed points as well as periodic points of period 1 .

Example (2): Consider the BCI-algebra Z of example (1) where the subset $A=\{0, r\}$ is a strongly invariant subset of $Z$ because $\Psi(A)=A$ while the subset $B=\{0, p\}$ is invariant because $\Psi(\mathrm{B}) \subset \mathrm{B}$.

\section{Main Theorems}

Theorem 5.1: In the discrete dynamical system $(Z, \Psi)$ the set of all fixed points in $Z$ is a BCI-subalgebra of $Z$.

Proof: Let $Z$ be a BCI-algebra and a mapping $\Psi: Z \rightarrow Z$ is a homomorphism. Suppose that $Z_{f}$ be the set of all fixed points in $Z_{\text {. We show that }} Z_{f}$ is a BCI-subalgebra for this $Z_{f}$ has to satisfy the conditions of BCI-subalgebra. Since $\Psi$ is a homomorphism therefore by proposition 4.1 we have $\Psi(0)=0$ which implies that 0 is a fixed point

$\Longrightarrow 0 \in Z_{f} \Rightarrow Z_{f}$ is a non-vacuous set.

Thus condition (s-i) of BCI-subalgebra holds in $Z_{f}$. Now assume that $a, b \in Z_{f}$ then

$$
\Psi(\mathrm{a})=\mathrm{a}
$$

And

$$
\Psi(\mathrm{b})=\mathrm{b}
$$

Since $\Psi$ is a homomorphism therefore we get

$$
\Psi(\mathrm{a} * \mathrm{~b})=\Psi(\mathrm{a}) * \Psi(\mathrm{b})
$$

Using (3) and (4) in (5) we get

$\Psi(\mathrm{a} * \mathrm{~b})=\mathrm{a} * \mathrm{~b} \because \mathrm{a}$ and $\mathrm{b}$ are fixed points

$\Longrightarrow \mathrm{a} * \mathrm{~b}$ is a fixed point

$\Rightarrow \mathrm{a} * \mathrm{~b} \in Z_{f}$

Thus for any $a, b \in Z_{f}$ we have $a * b \in Z_{f}$

Hence $Z_{f}$ is a BCI-subalgebra.

Theorem 5.2: In the discrete dynamical system $(Z, \Psi)$ the set of all periodic points in $Z$ is a BCI-subalgebra of $Z$.

Proof: Let $Z$ be a BCI-algebra and a mapping $\Psi: Z \rightarrow Z$ is a homomorphism, suppose that $Z_{p}$ be the set of all periodic points in BCI-algebra we show that $Z_{p}$ is a $\mathrm{BCI}$-subalgebra for this $Z_{p}$ has to satisfy the conditions of definition 2.3. Since $\Psi$ is a homomorphism therefore by proposition 4.1 . We have $\Psi(0)=0 \Longrightarrow 0$ is a periodic point of a period $1 \Rightarrow 0 \in Z_{p}$ $\Rightarrow Z_{p} \neq\{\}$ thus condition (s-i) of definition 2.3 holds in $Z_{p}$.

Let $a, b \in Z_{p}$ and suppose that the periods of ' $a$ ' and ' $b$ ' are $m$ and $n$ respectively. 
Such that

$$
\Psi^{m}(\mathrm{a})=\mathrm{a}
$$

And

$$
\Psi^{n}(\mathrm{~b})=\mathrm{b}
$$

Here we take $\mathrm{r}=\mathrm{LCM}[\mathrm{m}, \mathrm{n}]$ then by proposition 4.3 equations (6) and (7) becomes

$$
\Psi^{r}(\mathrm{a})=\mathrm{a}
$$

And

$$
\Psi^{r}(\mathrm{~b})=\mathrm{b}
$$

Now consider

$$
\Psi^{r}(\mathrm{a} * \mathrm{~b})=\Psi^{r}(\mathrm{a}) * \Psi^{r}(\mathrm{~b}) \because \text { proposition } 4.2
$$

Using equations (8) and (9) in (10) we get $\Psi^{r}(\mathrm{a} * \mathrm{~b})=\mathrm{a} * \mathrm{~b}$ $\Rightarrow \mathrm{a} * \mathrm{~b}$ is a periodic point of period " $\mathrm{r}$ " $\Rightarrow \mathrm{a} * \mathrm{~b} \in \mathrm{Z}_{p}$ Thus for any $\mathrm{a}, \mathrm{b} \in \mathrm{Z}_{p}$ we have $\mathrm{a} * \mathrm{~b} \in \mathrm{Z}_{p}$

Hence $p$ is a BCI-subalgebra

Theorem 5.3: Let $(Z, \Psi)$ is a discrete dynamical system then the set of all fixed points in $Z$ is a strongly invariant ( $S$ invariant) subset of BCI-algebra $Z$.

Proof: Let $Z_{f}$ be the set of all fixed points in $Z$

Let $\Psi$ (a) $\in \Psi\left(Z_{f}\right)$ where "a" is any element of $Z$

$\Longrightarrow \Psi(\mathrm{a})=\mathrm{a} \because \Psi\left(Z_{f}\right)$ is a set of the images of all the fixed points

$$
\begin{aligned}
& \Rightarrow \mathrm{a} \in Z_{f} \\
& \Rightarrow \Psi(a) \in Z_{f} \because \Psi(a)=a \\
& \text { Thus } \Psi(a) \in \Psi\left(Z_{f}\right) \Rightarrow \Psi(a) \in Z_{f}
\end{aligned}
$$

$$
\text { Therefore we have } \Psi\left(Z_{f}\right) \subseteq Z_{f}
$$

Now we suppose that $\mathrm{a} \in \mathrm{Z}_{f}$

$$
\Longrightarrow \Psi(\mathrm{a})=\mathrm{a}
$$

$\because Z_{f}$ is a set of fixed points where $\Psi(a) \in \Psi\left(Z_{f}\right)$

$\Rightarrow \mathrm{a} \in \Psi\left(Z_{f}\right) \because$ using (12)

Thus $\mathrm{a} \in \mathrm{Z}_{f} \Rightarrow \mathrm{a} \in \Psi\left(\mathrm{Z}_{f}\right)$

$$
\text { Therefore we have } Z_{f} \subseteq \Psi\left(Z_{f}\right)
$$

From (11) and (13) we have $\Psi\left(Z_{f}\right)=Z_{f}$

Hence $Z_{f}$ in $Z$ is $\mathrm{S}$-invariant or strongly invariant.

Theorem 5.4: In the discrete dynamical system $(Z, \Psi)$ if "a" is a fixed point and " $b$ " is a periodic point in $Z$ then $a * b$ is a periodic point in $\mathrm{Z}$.

Proof: Since "a" is a fixed point therefore we have

$$
\Psi(\mathrm{a})=\mathrm{a}
$$

Let the period of the point " $b$ " is $\mathrm{k}$ such that

$$
\Psi^{k}(\mathrm{~b})=\mathrm{b}
$$

As " $a$ " is a fixed point of period 1 and 1 divides $k$ therefore by proposition 4.3 we have

$$
\Psi^{k}(\mathrm{a})=\mathrm{a}
$$

Now by using the homomorphism property of $\Psi^{k}$ we can get

$$
\Psi^{k}(\mathrm{a} * \mathrm{~b})=\Psi^{k}(\mathrm{a}) * \Psi^{k}(\mathrm{~b})
$$

Using (15) and (16) in (17) we get

$$
\Psi^{k}(\mathrm{a} * \mathrm{~b})=\mathrm{a} * \mathrm{~b}
$$

Equation (18) implies that the period of $a * b$ is $k$ hence $a * b$ is a periodic point.

Theorem 5.5: In the discrete dynamical system $(Z, \Psi)$ if $A$ is a subset of $Z$ such that $\Psi(Z) \subset A \subset Z$, then $\mathrm{A}$ is invariant with respect to $\Psi$.

Proof: Since we have

$$
\Psi(\mathrm{Z}) \subset A \subset \mathrm{Z}
$$

From (19) we have

$$
A \subset \text { Z }
$$

And

$$
\Psi(\mathrm{Z}) \subset A
$$

From (20) we can get

$$
\Psi(A) \subset \Psi(\text { Z) }
$$

From (21) and (22) we get

$$
\Psi(A) \subset \Psi(\text { Z }) \subset A
$$

Equation (23) Implies that $\Psi(A) \subset A$

Hence $A$ is an invariant subset of BCI-algebra $Z$,

Example 3: Consider the BCI-algebra $Z=\{0, \mathrm{p}, \mathrm{q}, \mathrm{r}\}$ and a mapping $\Psi: Z \rightarrow Z$ where the mapping is a homomorphism defined by $\Psi(0)=0, \Psi(\mathrm{p})=0, \Psi(\mathrm{q})=0$, and $\Psi(\mathrm{r})=\mathrm{r}$. let the subset of $Z$ is $A=\{0, p, r\}$ while $\Psi(Z)=\{0, r\}$ then it is clear that $\Psi(Z) \subset A \subset Z$. Hence $A$ is an invariant set of $Z$.

Theorem 5.6: In the discrete dynamical system $(Z, \Psi)$ if $Z$ is a p-semisimple BCI-algebra then the set of all the fixed points in $Z$ is an ideal $Z$.

Proof: Since Z is a p-semisimple BCI-algebra and a mapping $\Psi: Z \rightarrow Z$ is a homomorphism. Suppose that $Z_{f}$ is the set of all fixed points in $Z$. We show that $Z_{f}$ is an ideal of $Z$ for this $Z_{f}$ has to satisfy the conditions of definition 2.4. Since $\Psi$ is a homomorphism therefore by proposition 4.1 we have $\Psi(0)=0 \Rightarrow 0 \in Z_{f} \Rightarrow Z_{f} \neq\{\}$. Thus the first condition of ideal holds in $Z_{f}$

Now we assume that $\mathrm{a} * \mathrm{~b} \in Z_{f}$ and $\mathrm{b} \in Z_{f}$

Then we have

$$
\Psi(\mathrm{a} * \mathrm{~b})=\mathrm{a} * \mathrm{~b}
$$




$$
\Psi(\mathrm{b})=\mathrm{b}
$$

We know that $\Psi$ is a homomorphism i.e., $\Psi$ $(a * b)=\Psi(a) * \Psi(b)$ so using this in (24) we get

$$
\Psi(\mathrm{a}) * \Psi(\mathrm{b})=\mathrm{a} * \mathrm{~b}
$$

Using (25) in (26) we get

$$
\Psi(\mathrm{a}) * \mathrm{~b}=\mathrm{a} * \mathrm{~b}
$$

By theorem $2.8\left(\mathrm{~T}_{2}{ }_{2}\right)$ we get $\Psi(\mathrm{a})=\mathrm{a} \Rightarrow \mathrm{a} \in \mathrm{Z}_{f}$

Thus $\mathrm{a} * \mathrm{~b} \in Z_{f}$ and $\mathrm{b} \in Z_{f} \Rightarrow \mathrm{a} \in Z_{f}$

Hence $Z_{f}$ is an ideal of p-semisimple BCI-algebra $\mathbf{Z}$

Theorem 5.7: In the discrete dynamical system $(Z, \Psi)$ if $Z$ is a p-semisimple BCI-algebra then the set that contains all the periodic points in $Z$ is an ideal of $Z$.

Proof: Since $Z$ is a p-semisimple BCI-algebra and a mapping $\Psi: Z \rightarrow Z$ is a homomorphism. Suppose that $Z_{p}$ be the set of all periodic points in $Z$. We show that $Z_{p}$ is an ideal of $Z$ for this $Z_{p}$ has to satisfy the conditions of definition 2.4. since $\Psi$ is a homomorphism therefore by proposition 4.1 we have $\Psi(0)=0$ that implies that 0 is a periodic point of period 1 $\Rightarrow 0 \in Z_{p} \Rightarrow Z_{p} \neq\{\}$. thus the first condition of ideal holds in $Z_{p}$.

Now we assume that $a * b \in Z_{p}$ and $b \in Z_{p}$ with periods $m$ and $n$ respectively

Then we have

$$
\Psi^{m}(\mathrm{a} * \mathrm{~b})=\mathrm{a} * \mathrm{~b}
$$

And

$$
\Psi^{n}(\mathrm{~b})=\mathrm{b}
$$

Here we take $\mathrm{r}=\mathrm{LCM}[\mathrm{m}, \mathrm{n}]$ then by proposition 4.3 equations (28) and (29) become

$$
\Psi^{r}(\mathrm{a} * \mathrm{~b})=\mathrm{a} * \mathrm{~b}
$$

And

$$
\Psi^{r}(\mathrm{~b})=\mathrm{b}
$$

By proposition 4.2 equation (30) becomes

$$
\Psi^{r}(\mathrm{a}) * \Psi^{r}(\mathrm{~b})=\mathrm{a} * \mathrm{~b}
$$

$\because \Psi^{r}$ is homomorphism

Using (31) in (32) we get

$$
\Psi^{r}(\mathrm{a}) * \mathrm{~b}=\mathrm{a} * \mathrm{~b}
$$

By theorem $2.8\left(\mathrm{~T}_{2}{ }_{2}\right)$ equation (33) becomes $\Psi^{r}(\mathrm{a})=\mathrm{a}$ $\Rightarrow$ 'a' is a periodic point of period ' $r$ ' $\Rightarrow a \in Z_{p}$.

Thus $\mathrm{a} * \mathrm{~b} \in \mathrm{Z}_{p}$ and $\mathrm{b} \in \mathrm{Z}_{p} \Rightarrow \mathrm{a} \in \mathrm{Z}_{p}$

Hence $Z_{p}$ is an ideal of p-semisimple BCI-algebra $\mathbf{Z}$

\section{Conclusion}

We see that discrete dynamical system with special properties plays a central role in the investigation of the structure of an algebraic system.

In this manuscript we introduce the concept of a discrete dynamical system $(Z, \Psi)$ in BCI-algebra, where $Z$ is a BCIalgebra and $\Psi$ is a homomorphism from $Z$ to $Z$ and establish some of their related properties. We prove that the sets of all periodic and fixed points in BCI-algebra $Z$ are the BCIsubalgebras. We show that when a subset of a BCI-algebra $Z$ is invariant with respect to $\Psi$. we prove that the sets of all fixed points and periodic points in p-semisimple BCI-algebra $Z$ are the ideals $Z$. We also prove that the set of all fixed points in Z is a S-invariant subset of a BCI-algebra Z.

The forthcoming study of discrete dynamical system in BCI-algebras may be the following topics are worth to be taken into account.

To describe other classes of BCI-algebra by using this concept.

To refer this concept to some other algebraic structures.

To consider the results of this concept to some possible applications in information systems and computer sciences.

\section{References}

[1] Fernandes, S., Ramos, C., Thapa, G., Lopes, L., \& Grácio, C. (2018). Discrete Dynamical Systems: A Brief Survey. Journal of the Institute of Engineering, 14 (1), 35-51.

[2] Bunder, M. W. (1998). Cancellation laws for BCI-algebra, atoms and p-semisimple BCI-algebras.". scientiae mathematicae japonicae, 1 (1), 19-22.

[3] Devaney, R. L. (1989). chaotic dynamical systems (second edition). Addison-wesley.

[4] Dardano, U., Dikranjan, D., \& Rinauro, S. (2017). Inertial properties in groups. arXiv preprint arXiv: 1705.02954.

[5] Dikranjan, D., \& Bruno, A. G. (2013). Discrete Dynamical systems in group theory. arXiv preprint arXiv: 1308.4035 .

[6] Gamelin, T. W. (1996). A History of Complex Dynamics, from Schroder to Fatou and Julia. By Daniel S. Alexander. Wiesbaden (Vieweg). Historia Mathematica, 23 (1), 74-86.

[7] Holms, J. P. (1990). Poincare, celestial mechanics, dynamicalsystems theory and chaos. PhysicsReports 193.3, 137-163.

[8] Hoo, C. S. (1988). A survey of BCK and BCI-algebras. Southeast Asian Bull. Math., 12.

[9] Iseki, K. (1966). An algebra related with a propositional calculus. proc. japan. Acad 42, 26-29.

[10] Iseki, k. (1980). On BCI-algebras. Math. Semi notes (presently Kobe. J. Math.) 8.

[11] Iseki, K., \& Tanaka, S. (1978). An introduction to the theory of BCK-algebras. Math. japonica, 23, 1-26.

[12] Meng, J., \& Jun, B. Y. (1994). BCK-algebras. Kyung Moon Sa Co., Seoul, Korea.

[13] Meng, J., \& Liu, Y. L. (2001). An introduction to BCI-algebras. (Chinese), Shanxi Scientific and Technological press, Xi'an, chaina. 
[14] Lei, T., \& Xi, C. (1985). p-Radical in BCI-algebras. Math. Japonica 30, 511-517.

[15] Lui, E. M. (2020). Structural Stability. Structural Engineering and Geomechanics-Volume 1, 198.

[16] Prior, A. N. (1962). Formal Logic, second edition. Oxford.

[17] De Vries, J. (2013). Elements of topological dynamics (Vol. 257). Springer Science \& Business Media.
[18] Silva, C. E. (2007). Invitation to ergodic theory (Vol. 42). American Mathematical Society.

[19] Smale, S. (1960). Morse inequalities for dynamical system. Bulletin of the American Mathematical society, 66 (1), 43-49.

[20] Dikranjan, D., \& Bruno, A. G. (2016). Entropy on abelian groups. Advances in Mathematics, 298, 612-653. 